\title{
Phased Human Activity Recognition based on GPS
}

\author{
Ryoichi Sekiguchi \\ Tokyo Denki University \\ 5 Senju-Asahi-cho, Adachi-ku \\ Tokyo 120-8551, Japan \\ 20jkm17@ms.dendai.ac.jp \\ Masayasu Kumano \\ Tokyo Denki University \\ 5 Senju-Asahi-cho, Adachi-ku \\ Tokyo 120-8551, Japan \\ 21amj09@ms.dendai.ac.jp
}

\author{
Kenji Abe \\ Tokyo Denki University \\ 5 Senju-Asahi-cho, Adachi-ku \\ Tokyo 120-8551, Japan \\ 20jkm02@ms.dendai.ac.jp \\ Daisuke Asakura \\ Tokyo Denki University \\ 5 Senju-Asahi-cho, Adachi-ku \\ Tokyo 120-8551, Japan \\ 18aj004@ms.dendai.ac.jp
}

\author{
Shogo Suzuki \\ Tokyo Denki University \\ 5 Senju-Asahi-cho, Adachi-ku \\ Tokyo 120-8551, Japan \\ 20jkm16@ms.dendai.ac.jp \\ Ryo Okabe \\ Tokyo Denki University \\ 5 Senju-Asahi-cho, Adachi-ku \\ Tokyo 120-8551, Japan \\ 18aj037@ms.dendai.ac.jp
}

\author{
Takeru Kariya \\ Tokyo Denki University \\ 5 Senju-Asahi-cho, Adachi-ku \\ Tokyo 120-8551, Japan \\ 18aj046@ms.dendai.ac.jp
}

\author{
Masaki Kawakatsu \\ Tokyo Denki University \\ 5 Senju-Asahi-cho, Adachi-ku \\ Tokyo 120-8551, Japan \\ kawakatu@mail.dendai.ac.jp
}

\begin{abstract}
This paper describes an activity recognition method for SussexHuawei Locomotion-Transportation (SHL) recognition challenge by team TDU_BSA_BCI. The classification accuracy has been improved by switching the estimation model, depending on whether the location is available. Data, including location were classified by Deep Neural Network including LSTM layer. Data that exclude location were classified by the Gradient Boosting Decision Tree. The 2 outputs have been combined. They were optimized by applying a median filter. In the submission phase, the best F-measure obtained for the SHL validation-set was $65 \%$.
\end{abstract}

\section{CCS CONCEPTS}

- Computing methodologies $\rightarrow$ Activity recognition and understanding.

\section{KEYWORDS}

Classification Flow; LSTM; Gradient Boosting Decision Tree, Median Filter

\section{ACM Reference Format:}

Ryoichi Sekiguchi, Kenji Abe, Shogo Suzuki, Masayasu Kumano, Daisuke Asakura, Ryo Okabe, Takeru Kariya, and Masaki Kawakatsu. 2021. Phased Human Activity Recognition based on GPS. In Adjunct Proceedings of the 2021 ACM International foint Conference on Pervasive and Ubiquitous Computing and Proceedings of the 2021 ACM International Symposium on Wearable Computers (UbiComp-ISWC '21 Adjunct), September 21-26, 2021, Virtual, USA. ACM, New York, NY, USA, 5 pages. https://doi.org/10.1145/3460418.3479382

Permission to make digital or hard copies of part or all of this work for personal or classroom use is granted without fee provided that copies are not made or distributed for profit or commercial advantage and that copies bear this notice and the full citation on the first page. Copyrights for third-party components of this work must be honored. For all other uses, contact the owner/author(s).

UbiComp-ISWC '21 Adjunct, September 21-26, 2021, Virtual, USA

(C) 2021 Copyright held by the owner/author(s).

ACM ISBN 978-1-4503-8461-2/21/09.

https://doi.org/10.1145/3460418.3479382

\section{INTRODUCTION}

Many studies are attempting to estimate the movement state using the smartphone's sensor. We participated in Sussex-Huawei Locomotion-Transportation (SHL) recognition challenge as the team TDU_BSA_BCI. Our target is developing the movement state classification algorithm using the SHL dataset 2021 [1]. We participated in the SHL recognition challenge with the team TDU_BSA in 2020 [2]. Accelerometers, geomagnetic sensors, gyro sensors, and barometric pressure sensors cannot be used in this challenge. It is necessary to consider a method appropriate for GPS, Wi-Fi, and Cell that can be used in this challenge. [5] shows activity recognition using GPS. [5] does not consider the case where the current position cannot be obtained from GPS. For improving classification accuracy, we changed the model which is used to determine whether it has the location information or not. It was changed to Deep Learning if location information is included, and eXtream Gradient Boosting (XGBoost) if it is not included. This method, shown in [4], was effective in improving the estimation accuracy.

\section{SHL CHALLENGE DATA}

The data are divided into 3 parts: train, validate and test. The data comprise 59 days of the training set, 4 days of the validation set and 39 days of the testing set. The training set is collected by User1. The validation set is a mixture of User2 and User3. The testing set is a mixture of User2 and User3. These three data are collected at Hips. The SHL Challenge data set in 2021 has Location, GPS, Wi-Fi, Cells, and Label. Location, GPS, Wi-Fi, and Cells are collected at $1 \mathrm{~Hz}$. In the validation set, there is a day that Wi-Fi is not collected.

The location has the accuracy of this location, Latitude, and Longitude. GPS has Satellite ID, Signal-to-noise ratio, Azimuth, and Elevation. Wi-Fi has Basic Service Set Identifier (BSSID), Service Set Identifier (SSID), Received Signal Strength Indicator (RSSI), Frequency, and Capabilities. Cells have a type of cell: LTE, WCDMA or GSM, status of the connection of the phone to this cell, Cell identity, Mobile Country Code, Mobile Network Code, Physical 
Cell Id, Tracking Area Code, and Signal Strength. Furthermore, the label has activity labels: "Still," "Walking," "Run," "Bike," "Car," "Bus," "Train," and "Subway," corresponding to each epoch time.

This challenge recognizes labels in the testing set.

\section{PREPROCESSING}

The epoch time of each sensor data and labeled data in the dataset weren't synchronized. We synchronized the epoch times of each sensor data and labeled data. We used the merge_asof function in Pandas for the synchronized of epoch times. We set the tolerance to $5 \mathrm{~s}$. One sensor data corresponds to one label data with the closest epoch time within $5 \mathrm{~s}$ before and after. We filled the sensor data of the label data whose sensor data was not synchronized with $\mathrm{NaN}$.

To calculate the distance traveled in $1 \mathrm{~s}$ from the longitude and latitude we used the Hubeny formula first term as shown bellow. The geodetic system is the World Geodetic System (WGS84).

$$
D=\sqrt{(M \times d P)^{2}+(N \times \cos (P) \times d R)^{2}}
$$

D: distance between 2 points $(\mathrm{m})$

$\mathrm{P}$ : mean latitude from 2 points

$\mathrm{dP}$ : latitude difference between 2 points

$\mathrm{dR}$ : longitude difference between 2 points

M: radius of curvature

$\mathrm{N}$ : radius of prime vertical circle

Velocity was calculated from the distance calculated using Hubeny formula first term. The unit of Velocity is $[\mathrm{km} / \mathrm{h}]$. Since D is the distance traveled in 1 second, Velocity can be obtained by multiplying $\mathrm{D}$ by 3.6.

$V=D \times 3.6$

$\mathrm{V}$ : velocity $(\mathrm{km} / \mathrm{h})$

Angular acceleration was calculated from the azimuth angle. Angular Acceleration is the difference in azimuth angle.

$$
\begin{aligned}
& A A=\arctan \left(\frac{\sin (d R)}{\cos (L P) \times \tan (T P)-\sin (L P) \times \cos (d R)}\right) \\
& \text { AA: azimuth angle } \\
& \text { dR: longitude difference between } 2 \text { points } \\
& \text { LP: latitude of the starting point } \\
& \text { TP: latitude of the end point }
\end{aligned}
$$

\section{METHODS}

We classified these data into 8 activities using classification flow.

The data were first sorted to determine whether it contained any location information. If the latitude and longitude corresponding to the label data is $\mathrm{NaN}$, that data does not contain location information. We call it "no location data". No location data were classified by a model that estimates it into seven activities: "Still," "Walking," "Bike," "Car," "Bus," "Train," and "Subway." This model didn't estimate the activity "Run" because no location data in the Validation-set do not have "Run" data. Next, data including location information were classified into 8 activities with deep learning. $\mathrm{NaN}$ cannot be input to Deep Learning. Therefore, we classified no Location data by XGBoost using statistical features.

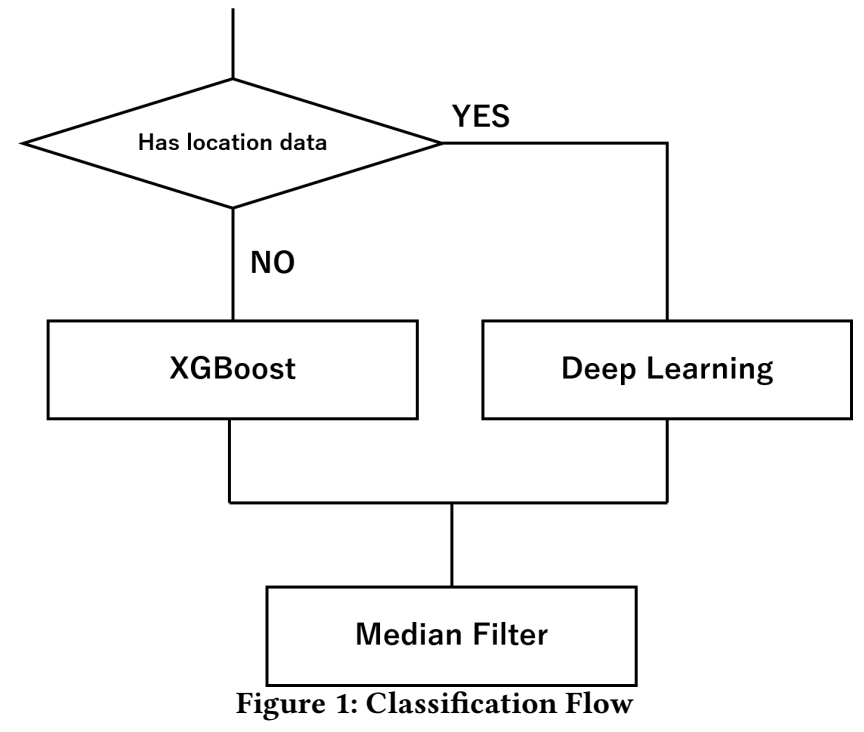

The Testing-set was classified in this flow, and we got an estimation. Figure 1 is the classification flow.

\subsection{No Location}

No location data were classified by a model that estimates it into 7 classes: "Still," "Walking," "Bike," "Car," "Bus," "Train," and "Subway." In this method, we used XGBoost. The following feature values were calculated every one second, then used classification. Figure 2 shows the confusion matrix in the validation-set data that exclude location information.

- The number of each cell (LTE, WCDMA, and GSM)

- Mean and valiance of each signal strength from the cell (LTE, WCDMA, and GSM)

- The number of received Wi-Fi access points

- Mean and standard deviation of Wi-Fi access point's RSSI. Calculated from each frequency

- Each $5 \mathrm{MHz}$ from $2412 \mathrm{MHz}$ to $2472 \mathrm{MHz}$

- Each $5 \mathrm{MHz}$ from $5180 \mathrm{MHz}$ to $5320 \mathrm{MHz}$

- Each $5 \mathrm{MHz}$ from $5500 \mathrm{MHz}$ to $5700 \mathrm{MHz}$

- The number of SSID from $29 \mathrm{Wi}-\mathrm{Fi}$ access points that is received high frequency in the Training-set

- The number of Wi-Fi access point includes the text "free" in its SSID

- The number of Wi-Fi access point includes the text "hotel" in its SSID

- The number of Wi-Fi access point includes the text "restaurant" in its SSID

\subsection{Deep Learning}

To classify data with location information into eight activity labels we employed a Deep Neural Network (DNN) model with a LongTerm Short Memory (LSTM) layer. Feature values were velocity, acceleration, and angular acceleration for $100 \mathrm{~s}$ before including estimated epoch time calculated from longitude and latitude. If feature values were not acquired for $100 \mathrm{~s}$, and we centralized it 


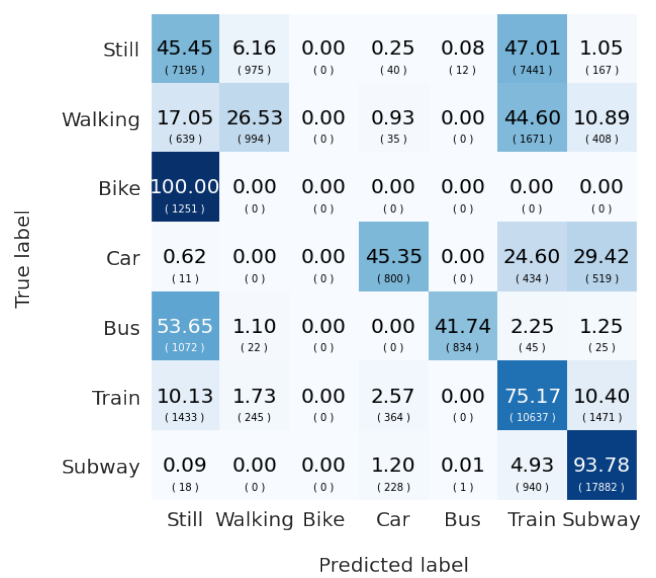

Figure 2: Confusion matrix of No Location (Data that does not include Location information of Validation-set)

with padding by zero. Figures 3, 4 and 5 show examples of velocity, acceleration and angular acceleration inputs.

The DNN is as follows. First, velocity, acceleration and angular acceleration are input to the $2 \mathrm{D}$ convolutional layer. The kernel size of the convolutional layer is $(1,3)$. This layer weights the three features and outputs the values to be input to the LSTM layer. Then, they were input to the LSTM layer. After that, they are input to 5 fully connected layers and get the output probabilities of 8 activities as output. Rectified Linear Unit (ReLU) was applied to the output of each layer other than the output layer. Softmax function was applied to the output. Figure 6 shows the DNN architecture.

Figure 7 shows the confusion matrix in the validation-set data that include location information.

\subsection{Post-processing}

We applied a median filter to estimate from the combined output of two model outputs. The filter length is $101 \mathrm{~s}$. If epoch time has a time gap of more than one second, we split that data and applied it to each data. By applying a median filter to the output, erroneous outputs that appear fragmentarily during the same continuous output can be corrected. The output is corrected more accurately by slicing at the part with the time gap. By using this process, the F1-score of the Validation-set was improved from $63 \%$ to $65 \%$. Figure 8 shows the confusion matrix before applying the median filter, and Figure 9 shows the confusion matrix after applying the median filter.

\section{RESULTS}

The F1-score of the Validation-set data, including the location information classified by the deep learning model, was 58\% (8 classes). The F1-score of the Validation-set data exclude location information classified by the No Location model was $27 \%$ ( 7 classes). The F1-score of "Bike" of the data not including the location information was $0 \%$. However, the ratio of "Bike" data that exclude location information is about $10 \%$ of the total number of "Bike" data, so it did not significantly affect the F1-score of the entire Validation-set. The two outputs have been combined. By applying the median filter to

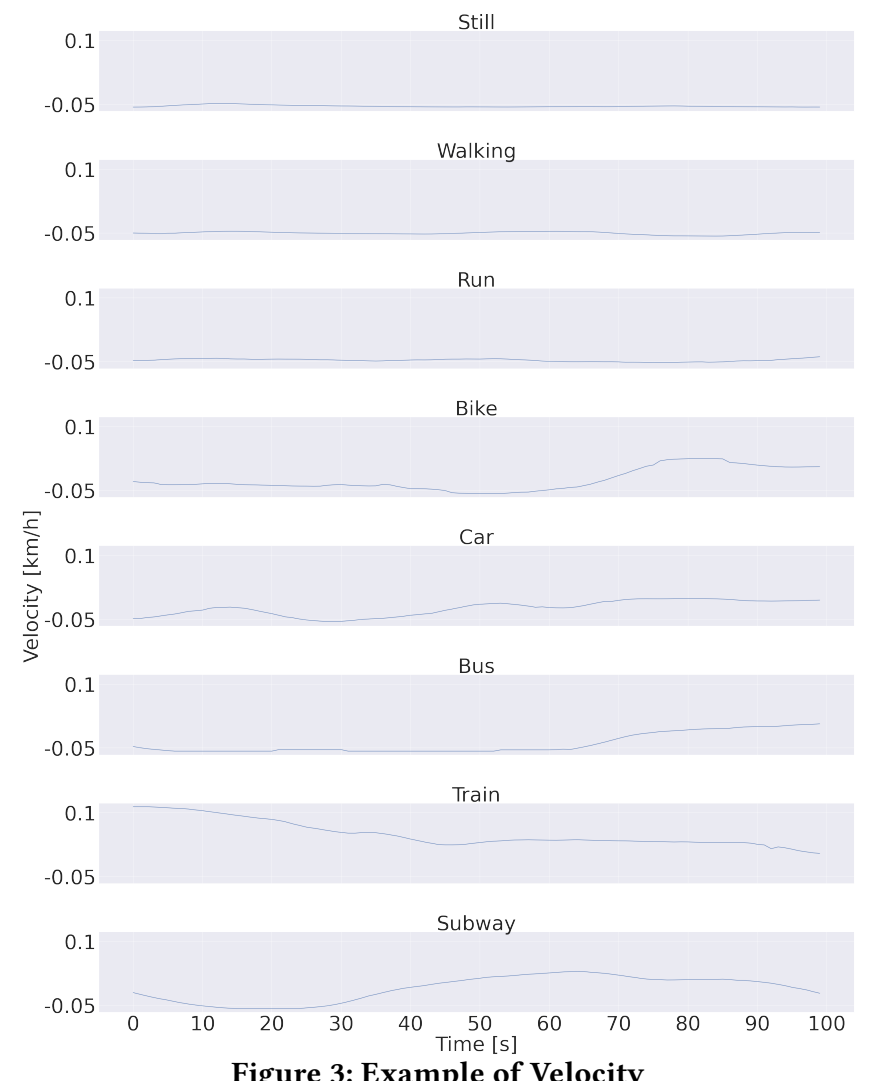

Figure 3: Example of Velocity

Table 1: The detail of the models created in this challenge

\begin{tabular}{ccl}
\hline Model names & Model size & Learning time \\
\hline \hline No Location & $1.5 \mathrm{MB}$ & 5 minutes \\
Deep Learning & $14.1 \mathrm{MB}$ & 5 minutes \\
\hline
\end{tabular}

them, we got the final estimation result. The final F1-score of The Validation-set was $65 \%$. These methods estimated the Test-set.

\section{CONCLUSION}

In this paper, we performed classification flow for activity recognition. The classification flow switched the estimation model used for classification depending on the availability of location. DNN including LSTM layer classified data, including location. XGBoost classifies data that exclude location. The outputs of the two models were combined, and we obtained the final estimation result by applying the median filter. In the final verification, the F1-score of the Validation-set was $65 \%$. The recognition result for the testing dataset will be presented in the summary paper of the challenge [3].

\section{COMPUTER RESOURCES}

CPU Ryzen9 3900XT, 3.8GHz, 12 core 24 threads, RAM 128GB, Geforce RTX 3080. Table 1 shows the learning time of the models created in this challenge. 


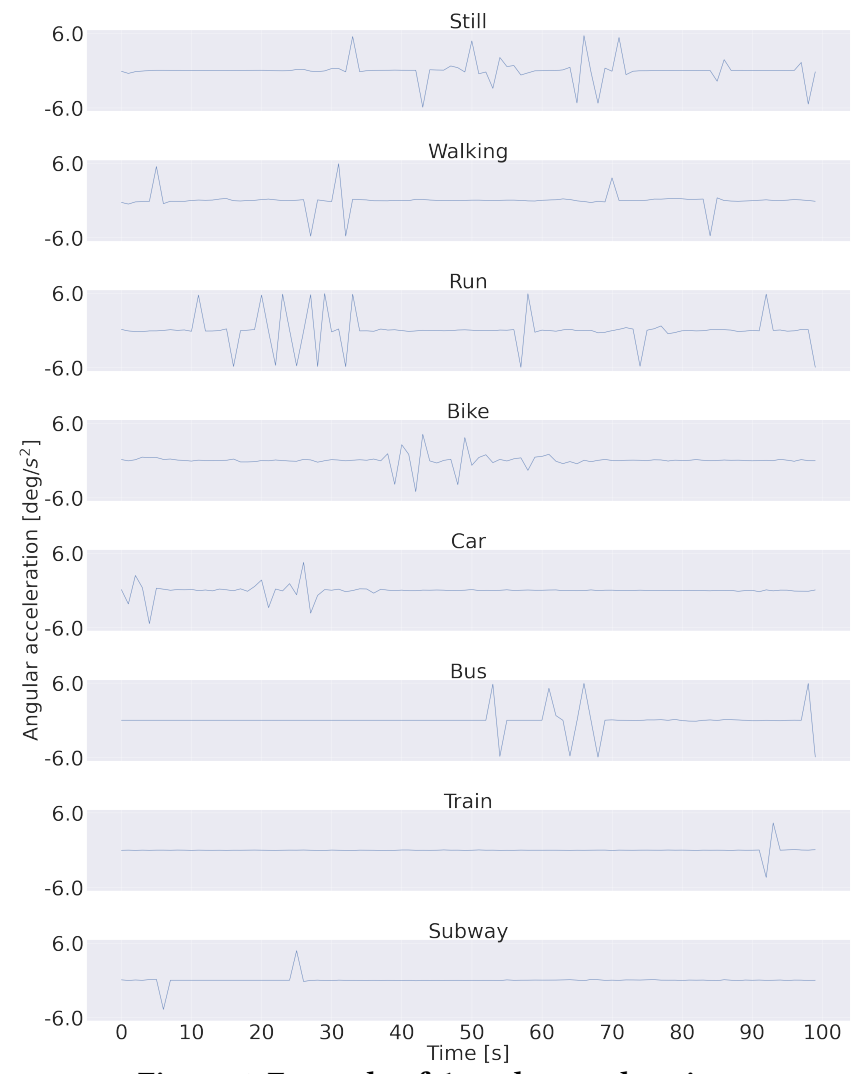

Figure 5: Example of Angular acceleration

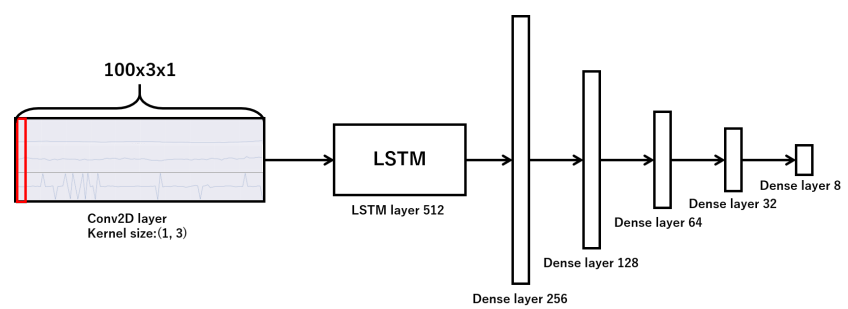

Figure 6: DNN model

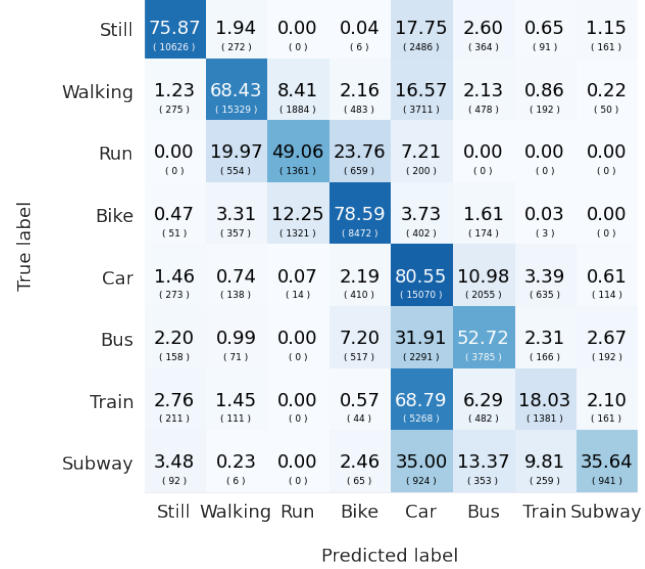

Figure 7: Confusion matrix of Deep Learning (Data including Location information of Validation-set)

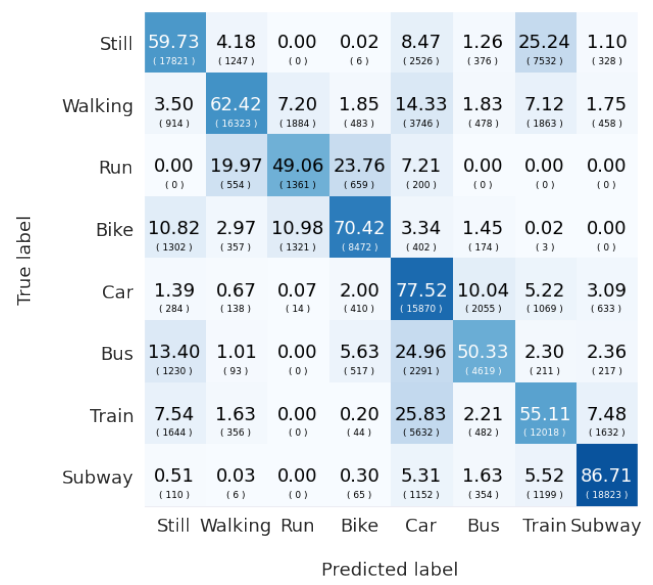

Figure 8: Confusion matrix of the classification flow (Validation-set)

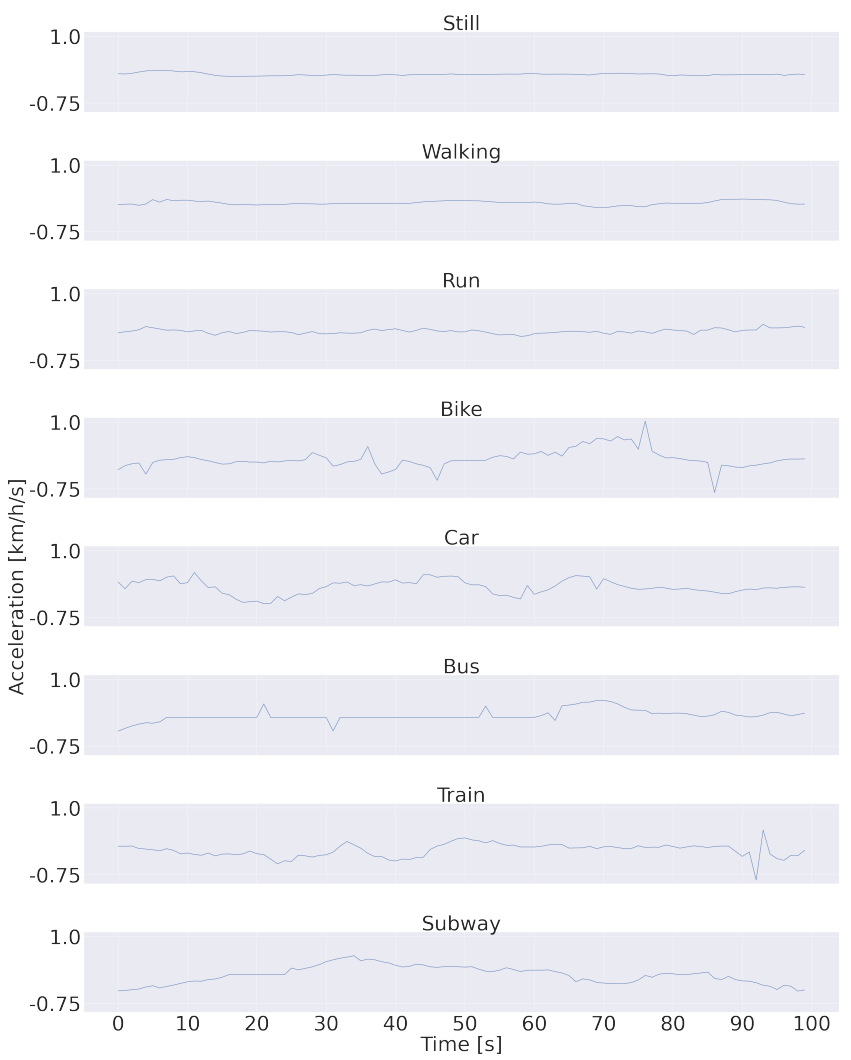

Figure 4: Example of Acceleration

\section{REFERENCES}

[1] H. Gjoreski, M. Ciliberto, L. Wang, F. J. Ordonez Morales, S. Mekki, S. Valentin, and D. Roggen. 2018. The University of Sussex-Huawei Locomotion and Transportation Dataset for Multimodal Analytics With Mobile Devices. IEEE Access 6 (2018), 42592-42604.

[2] Ryoichi Sekiguchi, Kenji Abe, Takumi Yokoyama, Masayasu Kumano, and Masaki Kawakatsu. 2020. Ensemble Learning for Human Activity Recognition. In $A d-$ junct Proceedings of the 2020 ACM International foint Conference on Pervasive and Ubiquitous Computing and Proceedings of the 2020 ACM International Symposium on Wearable Computers (Virtual Event, Mexico) (UbiComp-ISWC '20). 


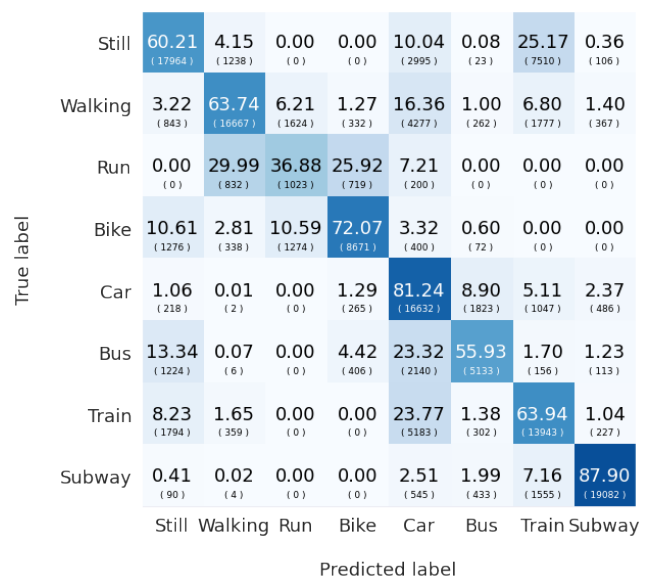

Figure 9: Confusion matrix of the median filter (Validationset)
Association for Computing Machinery, New York, NY, USA, 335-339. https: //doi.org/10.1145/3410530.3414346

[3] L. Wang, M. Ciliberto, H. Gjoreski, P. Lago, K. Murao, T. Okita, and D. Roggen. 2021. Locomotion and transportation Mode Recognition from GPS and radio signals: Summary of SHL Challenge 2021. Adjunct Proceedings of the 2021 ACM International foint Conference and Proceedings of the 2021 International Symposium on Pervasive and Ubiquitous Computing and Wearable Computers (2021).

[4] L. Wang, H. Gjoreski, M. Ciliberto, S. Mekki, S. Valentin, and D. Roggen. 2019. Enabling Reproducible Research in Sensor-Based Transportation Mode Recognition With the Sussex-Huawei Dataset. IEEE Access 7 (2019), 10870-10891.

[5] Yuki Watanabe, Reiji Suzumura, Shogo Matsuno, and Minoru Ohyama. 2019. Investigation of Context-aware System Using Activity Recognition. In 2019 International Conference on Artificial Intelligence in Information and Communication (ICAIIC). 287-291. https://doi.org/10.1109/ICAIIC.2019.8669035 